\title{
Education Platform at ZDM8
}

\author{
Jamie S. Lyman Gingerich,' Michael A. Pickart, and Chris Pierret ${ }^{3}$
}

\begin{abstract}
Interest among the zebrafish community in education and science accessibility for all ages has increased. At the 8th Annual Zebrafish Disease Models Conference (ZDM8), a specifically designed session enabled professional scientists, educators, and students to have a venue to present their science, discuss ideas in education, and partner to navigate a scientific meeting as an educational experience. This meeting report describes the format of the Platform Session as well as challenges and future plans to leverage impact of conferences on the local communities.
\end{abstract}

T He Education Platform at the 8th Annual Zebrafish Disease Models Conference (ZDM8) held in Boston, Massachusetts, from August 24 to August 27, 2015, provided an excellent backdrop to once again advance highly relevant professional science experiences for young investigators and other nontraditional scientists. Zebrafish Disease Models Society (ZDMS) co-chairs Drs. Chris Pierret (Mayo Clinic, Rochester, MN) and Michael Pickart (Concordia University Wisconsin, Mequon, WI) galvanized ZDMS members to leverage the rich resources of the conference for a large group of researchers, educators, and the newest generation of zebrafish scientists. All of the participants in the Education Platform, but particularly the middle and high school scientists, deeply appreciated the time and enthusiasm of the professionals who made the accomplishments (described hereunder) possible.

Those in the zebrafish community interested in education and science accessibility for all ages have steadily increased in number through several venues. This meeting had 50 or more participants in our workshop and interest group sessions. Similar efforts at the International Conference on Zebrafish Development and Genetics have also had 50 to as many as 100 participants. The education platform appears to give a voice to work not otherwise given space in our meeting venues.

For 2015, the overarching goals of the ZDM8 Education Platform were to help novice investigators navigate a scientific meeting, advance the scientific discoveries of nontraditional scientists, and expand the mentorship capacity of zebrafish scientists. These goals were easily achieved through poster presentations, listening to oral sessions, and interacting through mentored engagement with other scientists (Fig. 1). The more than 50 participants included educators, students, and professional scientists affiliated with groups including Project BioEyes (www.bioeyes.org), InSciEd Out (www .insciedout.org), Smith College Student Scientist Program, and Concordia University Wisconsin outreach. The participants presented eight posters during the regularly scheduled poster sessions of the conference. New to this meeting, nearly every conference speaker presenting during the scheduled Education Platform time volunteered 20-30 min to engage in postpresentation discussions with Education Platform participants.

The goal of creating an enriching environment for Education Platform participants by full integration at a professional scientific conference, such as ZDM8, is not without challenges. However, the organization and outcomes of this year's conference provide valuable lessons for achieving this ambitious goal for future venues. To overcome the prohibitive cost of full meeting attendance, a reduced rate was arranged for Education Platform participants during the third and fourth days of the conference. Shortly after their arrival, participants were guided through onboarding activities to prepare them for the planned conference events. For many, this was their first time in attendance at a professional conference, so it was important to orient them to this new environment. To facilitate the learning potential of attendance at scientific presentations, participants of all age levels and roles were assigned to integrated discussion groups led by mentors from the zebrafish scientific community, including graduate students, postdoctoral researchers, and faculty scientists.

Following orientation, the first session of the Education Platform began with the presentation of participants' science during the regularly scheduled poster session for the day and

\footnotetext{
${ }^{1}$ Department of Biology, University of Wisconsin-Eau Claire, Eau Claire, Wisconsin.

${ }^{2}$ School of Pharmacy, Concordia University Wisconsin, Mequon, Wisconsin.

${ }^{3}$ Department of Biochemistry and Molecular Biology, Mayo Clinic, Rochester, Minnesota.
}

(C) Jamie S. Lyman Gingerich, et al., 2016; Published by Mary Ann Liebert, Inc. This Open Access article is distributed under the terms of the Creative Commons Attribution Noncommercial License (http://creativecommons.org/licenses/by-nc/4.0/) which permits any noncommercial use, distribution, and reproduction in any medium, provided the original author(s) and the source are credited. 


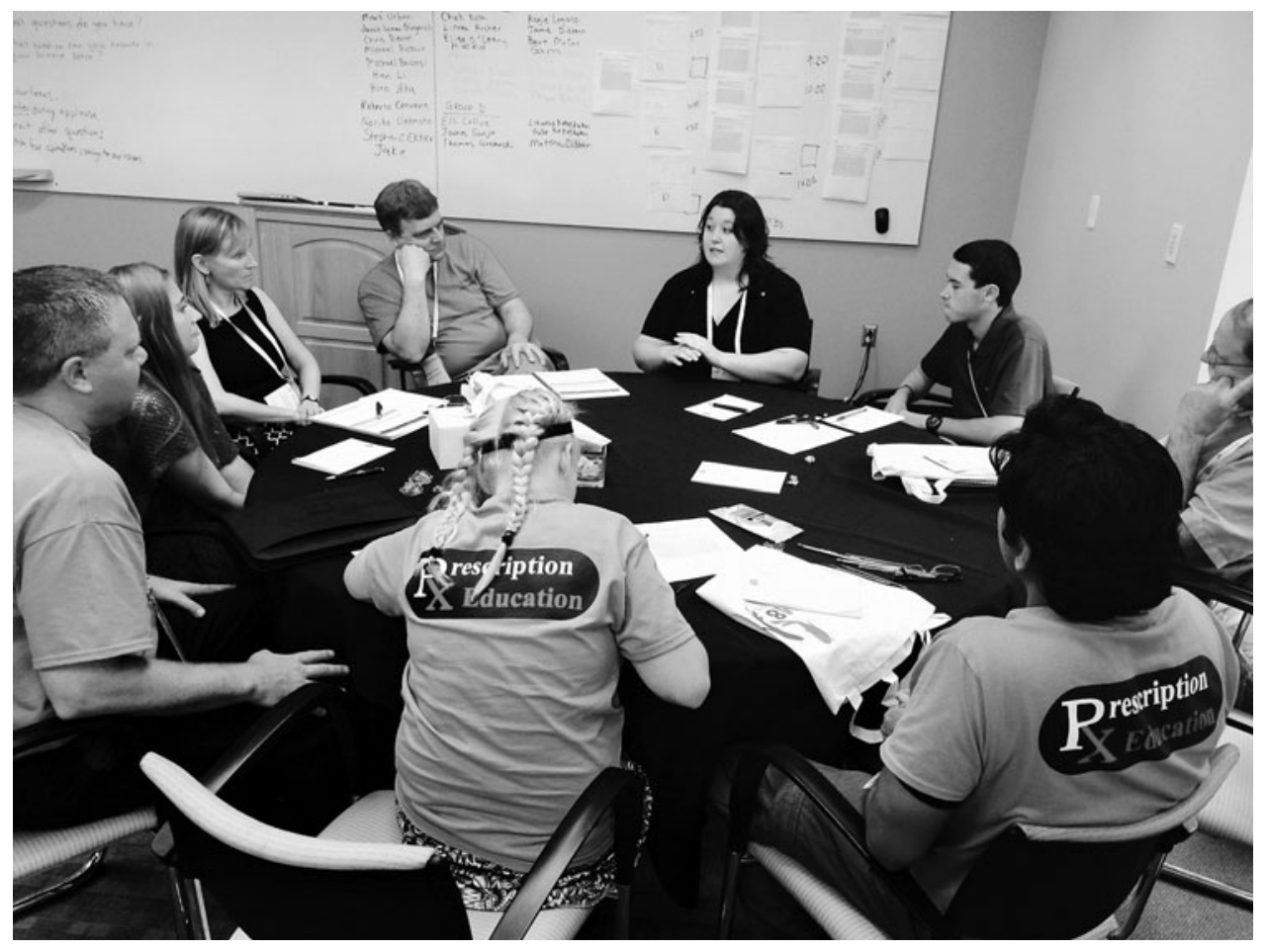

FIG. 1. Participants in the ZDM8 education platform engage in discussion with one of the platform presenters.

included young investigators (from elementary school, middle school, and high school). This work included a wide range of topics for investigation such as inexpensive technologies to alleviate the cost barrier to science in schools as well as research on using 3D protein modeling and printing technology to support entry of middle and high school students into molecular research using zebrafish. Additional posters introduced currently operating educational outreach programs to conference attendees, assessed the impact of the programs on student learning, and shared the development of new tools for authentic science experiences in the classroom. The Education Platform participants also used some of the poster session time to interact with other poster presenters to learn about new avenues of research.

The highlight of the Education Platform was mentored attendance at the oral scientific presentations. For this activity, participants attended talks with their mentor. Following all talks, students retired for a roundtable discussion that focused on the presented material. For the majority of talks, the presenters also met with the group (immediately or at a later time). Thanks to their participation, the mentored "meet the scientist"' opportunities were invaluable for furthering the connections and scientific development fostered during the oral sessions. During these discussions, participants were encouraged to revisit their own science and think about how knowledge gained at the poster session could be used to explore new paths in research.

Closing of the Education Platform included evaluation of the effectiveness of the meeting on participants' scientific growth (to be presented elsewhere) and discussion of ways to meet the challenges of advancing opportunities for nontraditional scientists. One clear challenge identified for future Education Platforms is increasing the involvement of educators, students, and business leaders local to the conference site to leverage the conference impact on the local community. Future plans include developing ways to encourage participation in scientific meetings by local community members, developing tools to effectively evaluate education programs, and continuing to increase the accessibility of zebrafish research. The next Education Platform is planned for the Allied Genetics Conference to be held in Orlando, Florida, in July 2016. Please contact Drs. Pierret or Pickart for opportunities to assist with the Education Platform planned for this upcoming meeting.

\section{Disclosure Statement}

No competing financial interests exist.

Address correspondence to:

Chris Pierret, $P h D$

Department of Biochemistry and Molecular Biology Mayo Clinic

200 1st Street SW

Guggenheim 13

Rochester, MN 55905

E-mail: pierret.christopher@mayo.edu 